\title{
Migraine associated with auditory-vestibular dysfunction
}

\section{Renato Cal', Fayez Bahmad Jr ${ }^{2}$}

Keywords: migraine, vestibulocochlear system, vertigo.

\section{Summary}

\begin{abstract}
$\mathrm{T}$
1 he association between hearing and balance disorders with migraine is known since the times of the ancient Greeks, when Aretaeus from Cappadocia in 131 B.C, made an accurate and detailed description of this occurrence during a migraine episode. We present a broad review of migraine neurotological manifestations, using the most recent publications associated with epidemiology, clinical presentation, pathophysiology, diagnostic methods and treatment for this syndrome. Aim: to describe the clinical entity: "Migraine associated with auditoryvestibular dysfunction" in order to help otorhinolaryngologists and neurologists in the diagnosis and management of such disorder. Final Remarks: There is a strong association between neurotological symptoms and migraine, and the auditory-vestibular dysfunction-associated migraine is the most common cause of spontaneous episodic vertigo (nonpositional). Symptoms may vary broadly among patients, making it a diagnostic challenge to the otorhinolaryngologist. This entity usually presents with positional or spontaneous vertigo spells, lasting for seconds or days, associated with migraine symptoms. A better understanding of the relationship between central vestibular mechanisms and migraine mechanisms, besides the discovery of ionic channel disorders in some cases of migraine, ataxia and vertigo, may lead to a better understanding of migraine pathophysiology associated with audio-vestibular disorder.
\end{abstract}

${ }^{1}$ Otorhinolaryngologist, researcher of the Otology Department, Massachusetts Eye \& Ear Infirmary.

${ }^{2}$ Otorhinolaryngologist, graduation doctoral student, UnB Medical School, assistant physician of the Otorhinolaryngology and Head \& Neck Surgery Department, Brasilia University Hospital, researcher of the Otology Department, Massachusetts Eye \& Ear Infirmary. Address for correspondence: Renato Cal - 243 Charles Street 4th Floor Room 468 Boston MA USA Zip Code 02114.

The author obtained funding from the CNPQ - MEC (Doctoral Scholarship) - Fayez Bahmad Jr.

This paper was submitted to the RBORL-SGP (Publishing Manager System) on 29 August 2006. code 3363. The article was accepted on 5 November 2006. 


\section{INTRODUCTION}

The association between hearing/balance disorders and migraine has been recognized since ancient Greece, when in 131 a.C., Aretaeus of Cappadocia described precisely and in detail the occurrence of both conditions during a migraine crisis. ${ }^{1}$ In 1861, Prosper Ménière published a classical paper describing the symptoms of Ménière Syndrome (MS) in migraine patients. ${ }^{2}$ In 1873 , Liveing reported a clear association between vertigo and migraine, which has since been also reported by various authors: Gowers et al., 1907; Symonds et al., 1926; Graham et al., 1968; and Kayan et al., 1984. These papers defined the concept that vertigo, hearing loss and tinnitus were part of the symptoms that some migraine patients presented. ${ }^{3-6}$

In 1961, Bickerstaff, introduced the Basilar Migraine concept, which is characterized by occipital headache with signs or symptoms of cranial nerve and/or brainstem dysfunction, such as: disorders of vision, vertigo, ataxia, speech disorders, tinnitus, and sensory alterations on extremities.

Various types of balance disorders have been reported during headache crises in migraine patients, such as rotational vertigo, positional vertigo, dizziness, intolerance to head movement, and other less common findings., ${ }^{48-16}$

Classical migraine patients presenting kinetosis in childhood or as adults appear to be more susceptible to migraine crises. ${ }^{4,16,-21}$.

Reported auditory disorders include phonophobia (intolerance to loud noise) and hyperacusis (abnormal noise sensitivity), first described by Tissot in 1778 (cited by Sachs et al., 1970), which may be related to headacheinduced stress. ${ }^{1}$

Currently, otologists and neurologists have received patients presenting a clinical picture of migraine-like headaches, episodes of dizziness, at times even vertigo, aural fullness, auditory symptoms and tinnitus. These patients have led to a description of a new clinical entity, which has been given various names, including: migraineassociated dizziness, migraine-related dizziness, migrainerelated vertigo, migrainous vertigo, migraine-anxiety related dizziness and migraine-associated cochleovestibular dysfunction. 6,14,20,22-24

Given the paucity of references on this theme in Portuguese, he authors have chosen the name "migrainerelated auditory-vestibular dysfunction" The reason for this is that many patients present vestibular findings that differ from the true concept of vertigo; symptoms may range from a feeling of unbalance to instability, vertigo, and in some cases even cochlear disorders such as tinnitus, intermittent dysacusis and auricular fullness.

The pathophysiology of migraine-related auditoryvestibular symptoms has not been fully clarified, but its clinical manifestation is varied. ${ }^{23}$ These patients usually pre- sent symptoms ranging from episodes of dizziness to acute vertigo and constant unbalance, sensorineural hearing loss, tinnitus, aural fullness and intermittent dysacusis. These symptoms are often mistaken with the classical symptoms of MS. It is, therefore, a syndrome that lies between migraine with aura and MS; the differential diagnosis between these three entities is often a major challenge (Figure 1 and Chart 1), requiring significant healthcare professional experience and knowledge about their clinical, diagnostic and therapeutic aspects. ${ }^{22,25-27}$

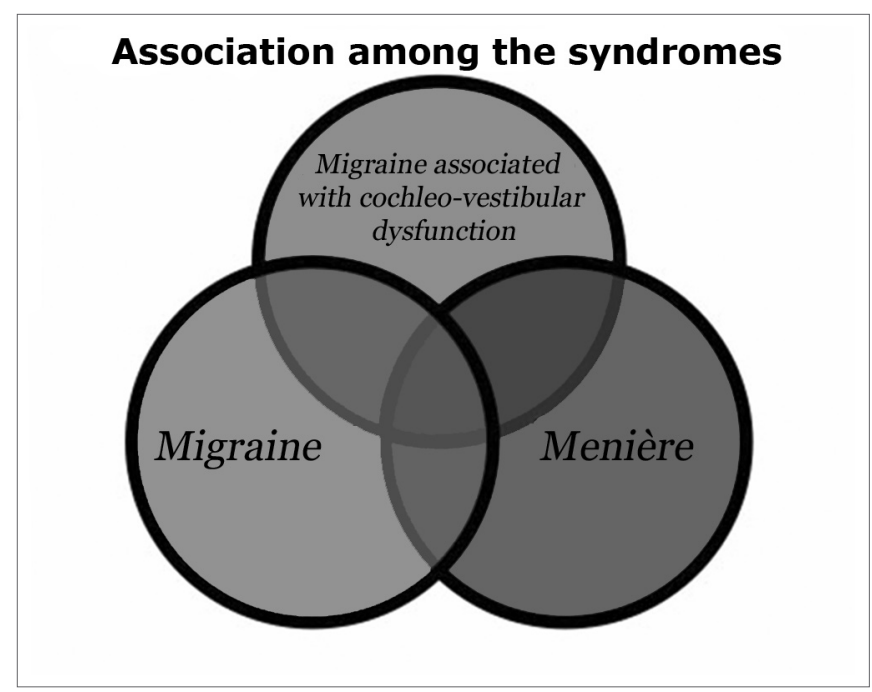

Figure 1. Relations among the syndromes.

\section{OBJECTIVES}

The purpose of this study was to describe the clinical entity "migraine-related auditory-vestibular dysfunction" to support otorhinolaryngologists and neurologists in the diagnosis and clinical management of this syndrome.

The authors reviewed the literature and the clinical experience of a North-American tertiary reference hospital for otoneurological disorders, looking at signs and symptoms and vestibular clinical findings for the diagnosis and treatment of these patients.

\section{Epidemiology}

Epidemiological data have underlined the increasing importance of migraine-related auditory-vestibular dysfunction in recent years. Recent papers have shown that migraine is one of the diseases with the highest morbidity worldwide; it affects about $4-6.5 \%$ of men and $11.2-18.2 \%$ of women both in the USA and in Europe. ${ }^{26,28} \mathrm{~A}$ correlation between migraine-related auditory-vestibular dysfunction and MS shows that the incidence of MS in the USA is about 5-15 for each 100,000 individuals (0.015\%). Based on these numbers, we may assume that if $1 \%$ of migraine-related auditory-vestibular dysfunction patients presented suffi- 
Chart 1. Association between symptoms and syndromes.

\begin{tabular}{|c|c|}
\hline Patients with Meniere's disease & Patients with migraine with aura \\
\hline Migraine symptoms & Meniere-like symptoms \\
\hline Headache & Vertigo and Floating Hearing Loss in the low frequencies \\
\hline Hinchcliffe et al, 1963 & Atkinson et al, 1962 \\
\hline Eklund et al, 1999 & Kayan et al, 1984 \\
\hline Ceranic et al, 2006 & Olsson et al, 1991 \\
\hline \multirow[t]{3}{*}{ Lopes et al, 2006} & Baloh et al, 1997 \& 2000 \\
\hline & Radtke et al, 2002 \\
\hline & Neuhauser et al, $2001 \& 2004$ \\
\hline Aura-like symptoms & Ear fullness and tinnitus \\
\hline Kentala et al, 1997 & Sand et al, 2000 \\
\hline \multirow[t]{3}{*}{ Baloh et al, 1999} & Baloh et al, 2000 \\
\hline & Radtke et al, 2002 \\
\hline & Neuhauser et al, $2001 \& 2004$ \\
\hline
\end{tabular}

cient episodes of vertigo to seek medical help, physicians would diagnose 15 times more patients with this entity compared to MS. ${ }^{25}$ The international medical literature pays insufficient attention to migraine-related auditory-vestibular dysfunction, suggesting that this condition is frequently not diagnosed. Lipton et al. (2002) studied a sample of 4,376 patients and revealed that migraine affects individuals in their most economically productive age group, namely $30-39$ years $(25.7 \%), 40-49$ years $(24.4 \%)$ and $18-29$ years (22.3\%), which underlies the importance of correctly diagnosing and treating this condition. ${ }^{28}$

This connection is even more evident in epidemiological studies of migraine patients: 28 to $36 \%$ of patients present associated dizziness, and 25 to $26 \%$ present vertigo. ${ }^{26}$ Similarly, $36 \%$ of patients that complain of dizziness may fit into the clinical criteria for migraine, and 61\% of patients with vertigo of unknown causes may also fit into the clinical criteria for migraine. ${ }^{24}$ In a case-control study of 10 patients, Furman et al. revealed that dizziness and vertigo occur in 54\% of migraine patients and in only $30 \%$ of patients with headaches of other origins, such as tension-type headaches, ${ }^{23,29}$ reinforcing the hypothesis of comorbidity between migraine and cochleovestibular disorders.

\section{Clinical and Diagnostic Findings}

Many individuals, and even healthcare professionals, still think that migraine is unilateral, pulsatile headache associated with visual and auditory disorders, and usually associated with nausea and vomiting. This concept remains correct and is the best know aspect of migraine with aura, according to the International Headache Society (IHS) criteria that were described in $1988 .{ }^{30}$ Currently, however, medical researchers have widened the scope of migraine, describing it as a global sensorial perceptive alteration in an individual. Any sensorial perception-related symptom may be described, including hearing, olfactive, visual, tactile, gustatory and postural disorders. ${ }^{6,23,28}$

\section{Differentiating migraine with aura from migraine with auditory-vestibular disorders}

Duration of headache is the first point to note when differentiating migraine-related auditory-vestibular dysfunction from classical migraine with aura. In classical migraine, symptoms may last from a few seconds to 60 minutes, while in migraine-related auditory-vestibular dysfunction cases, symptoms generally last hours, days or even months. Adult patients with migraine-associated vestibular dysfunction usually report spontaneous or positional vertigo, occasionally initiating as spontaneous vertigo and eventually becoming positional vertigo.6 An important point is that the duration of this type of positional vertigo differs from that seen in benign paroxysmal positional vertigo (BPPV). Patients with migraine-related cochleovestibular dysfunction usually describe vertigo while the head is in the due trigger position, contrary to what occurs in BPPV, in which vertigo lasts only a few seconds. Intolerance to movement is another characteristic feature of migraine-related auditory-vestibular dysfunction, which is very similar to the clinical findings of kinetosis (feeling of unbalance, illusion of movement and nausea worsened by head movements). ${ }^{31}$ Visual vertigo induced by movie screens, by lighting such as that found in stores and shopping malls and by computer screens is typical of migraine-related auditory-vestibular dysfunction.

Vertigo attacks may vary widely, lasting from minutes to two hours (46.4\%), and many hours or weeks $(30.4 \%) .^{22}$ Some individuals may require weeks to months to recover fully from an attack of vertigo. Such attacks may occur at intervals of days, weeks, months, or even 
years. Differentiating migraine-related auditory-vestibular dysfunction from migraine with aura may be more difficult when clinical findings last between 5 and 60 minutes,.

A careful clinical history is the best weapon for physicians to diagnose migraine-related auditory-vestibular dysfunction. Neuhauser et al published the clinical criteria for diagnosing this entity in a classical paper based on the IHS guidelines for diagnosing migraine. ${ }^{32}$ This paper, which has probably contributed most to the diagnostic standardization of that clinical entity, defined criteria for migraine-related vestibular disease for the defined and probable forms. These authors used the method of assessing three groups of patients: the first group included outpatient subjects presenting with vestibular disorders $(n=200)$, the second group included patients with migraine $(n=200)$ as defined by IHS criteria, and the third group included patients from the orthopedics unit of the same hospital that belonged to the same age group as group 1 patients (control group / $\mathrm{n}=200$ ). After analyzing these groups, the authors proposed the criteria for classifying migraine-related auditory-vestibular dysfunction that are presented on Chart 2.
These criteria have been adopted by most of the authors and international clinical units in this area in an attempt to standardize the diagnosis of migraine-related auditory-vestibular dysfunction. ${ }^{14-16,21-23,26,33,35}$ More recently, Marcus et al. developed a questionnaire based on Neuhauser's criteria to serve as a screening tool for patients with vestibular conditions in which a diagnosis of migraine-related vestibular disease is considered. ${ }^{33}$

Many authors have applied vestibular and auditory testing in patients diagnosed with migraine-related auditory-vestibular dysfunction in an attempt to establish a pattern for vestibular findings that would support the diagnosis of this condition. Another aim of vestibular testing would be to differentiate migraine-related auditory-vestibular dysfunction from the initial stages of MS. ${ }^{16,20,22,23,36,37,38}$

\section{Differentiating migraine-related auditory-vestibular dys- function from Ménière's syndrome (MS)}

Battista analyzed the audiometric findings of 76 patients with migraine-related auditory-vestibular dysfunction $\mathrm{n}$ and 34 patients diagnosed with MS. Results

Chart 2. Classification of Migraine associated with Vestibular-Auditory Dysfunction.

Classification of Migraine associated with Vestibular-Auditory Dysfunction.

\section{Established}

- Episodes of vestibular symptoms, of moderate to severe intensity (rotational vertigo, positional vertigo, other sensations of head movement intolerance)

- Migraine according to criteria from the International Headache Society, 2001.

- At least one of the following migraine symptoms during at least two dizziness spells: Headache, photophobia, phonophobia, visual disorders and other aura symptoms

- Ruling out other causes after clinical investigation

Probable

- Moderate to severe vestibular symptom spells (rotational vertigo, positional vertigo, other sensations of intolerance to head movement)

- According to the IHS, at least one of the following signs for migraine patients:

Migraine symptoms during at least two vertigo spells;

"Trigger" signs for migraine spells with some food stuff;

Sleep disorders;

Hormonal changes;

Positive response to anti-migraine drugs.

- Rule out other established cause of dizziness after due diagnostic investigation.

"Mild" vestibular symptoms were defined as those that do not interfere with the individual's daily activities; "moderate" when they interfere in daily activities, but do not prevent them, and "severe" when the individual is prevented from performing his/her daily activities.

Please bear in mind that non-vestibular dizziness, such as orthostatic hypotension, was not included. 
revealed that although there were descriptions in the literature of auditory disorders in patients with migrainerelated auditory-vestibular dysfunction, most of them had normal hearing, different from patients with MS (including intermittent hearing loss as part of the typical clinical picture of MS). ${ }^{22}$

Furman et al. studied patients with migraine-related auditory-vestibular dysfunction by using a battery of vestibular tests and found altered vestibular-spinal function tests in posturography. Balance was increased in patients with migraine-related auditory-vestibular dysfunction compared to both control groups, particularly in the Sensory Organization Test 4, 5 and 6 (Equitest ${ }^{\circledR}$ ); the same patients had normal oculomotor movements and caloric responses during vertigo-free periods, ${ }^{23,29}$ and more frequently altered rotatory chair test results compared to abnormal findings on electronystagmography (ENG). ${ }^{20,37}$

Dimitri et al. analyzed multiple variables in an attempt to establish a difference between migraine-related auditory-vestibular dysfunction and MS based on vestibular testing. The authors concluded that decreased vestibular responses in caloric testing and altered rotatory chair testing were significant in $91 \%$ of cases when differentiating migraine-related auditory-vestibular dysfunction and MS.38 In summary, there appears to be no typical pattern in vestibular testing for establishing a diagnosis of migrainerelated auditory-vestibular dysfunction, which reinforces even further the need for a carefully taken clinical history. Vestibular testing such as ENG, the rotatory chair test, electrocochleography (ECoG), and vestibular myogenic evoked potentials (VEMP) may support a diagnostic hypothesis raised in the clinical history. Progressive hearing loss is still the beset method for differentiating migraine-related auditory-vestibular dysfunction and MS.

\section{Treatment}

Having made a diagnosis of migraine-related auditory-vestibular dysfunction, the next step is counseling of patients. In general, patients with this condition respond positively to well-established therapy for migraine. ${ }^{21,39,40}$ The first step in controlling the symptoms is to persuade patients to adopt the so-called "migraine lifestyle," which includes dietary changes, regular exercise, and regular sleep. Dietary changes include decreasing or eliminating the use of aspartame, chocolate, caffeine and alcohol. ${ }^{40}$ It is also essential to exercise regularly, to decrease stress and to improve sleep. ${ }^{39}$ If these behavioral measures are unsuccessful, medication may be used for controlling vertigo episodes. There are the migraine-suppressing drugs (benzodiazepines, beta-blockers, tricyclic antidepressants, etc.) and the migraine-aborting drugs (mainly sumatriptan). Migraine-aborting drugs are an excellent option for treating migraine; however, in migraine-related auditoryvestibular symptoms, such drugs are generally not used because crises of vertigo usually occur as a headache aura. ${ }^{13}$ Thus, migraine-suppressing drugs are more used in otoneurology.

Possibly the most relevant data in the therapy of migraine-related auditory-vestibular symptoms were published in 2002 in a retrospective study that revealed that over $72 \%$ of patients improved or had complete remission of symptoms after step-by-step treatment was introduced. ${ }^{21}$ This treatment consists initially of changing the diet, followed by migraine-suppressing drugs such as low-dose tricyclic antidepressants, calcium channel blockers and beta-blockers.

The efficacy of drugs used in migraine-related auditory-vestibular dysfunction therapy is directly related with the ability of such drugs in combating or aborting headaches. ${ }^{13}$ Clinical randomized, prospective, double-blind, placebo-controlled studies for comparing the efficacy of various drugs in the treatment exclusively of migrainerelated auditory-vestibular symptoms have not yet been published in the literature. Thus, otoneurologists use their personal experience in managing this syndrome.

The authors emphasize the idea that, when managing correctly headaches associated with auditory-vestibular symptoms, physicians should be aware of side-effects and reactions to drugs that otorhinolaryngologists do not prescribe regularly in everyday practice. It is therefore wise to begin with the lowest pharmacological dose.

The first step in treatment is to start with behavioral measures such as an adequate diet, good sleeping habits, regular physical exercise, and a decrease in stress levels during at least one month. Drug therapy is initiated if patients continue with regular episodes of migraine-related auditory-vestibular symptoms. Preferred drugs are tricyclic antidepressants, especially nortryptiline $(10 \mathrm{mg} /$ day $)$ at night before sleeping, to minimize the main side effects (drowsiness and xerostomia). The initial dose should be maintained for two weeks, after which it is increased if migraine crises persist. Most of the patients benefit from a dose between 30 and $70 \mathrm{mg}$ /day without presenting side effects.

Beta-blockers are the second drugs of choice for the authors, especially propanolol. The initial dose is 40 $\mathrm{mg}$ /day; most patients end up taking around $80 \mathrm{mg} /$ day. It is important to note that most of the patients with a diagnosis of migraine-related auditory-vestibular dysfunction are young women; these patients tend to present arterial hypotension, which makes beta-blockers an added risk. It is clear that therapy for each patient should be approached individually, taking into account factors such as age, sex, comorbidities, etc., so that the lowest dose capable of controlling the disease may be used, thus decreasing significant side effects.

Reploeg et al. demonstrated that 100\% of migrainerelated auditory-vestibular dysfunction patients reported 
some improvement in vertigo and unbalance after initiating one of these therapies. ${ }^{21}$ Another study involving 53 migraine-related auditory-vestibular dysfunction patients revealed that the efficacy of drugs in controlling vestibular symptoms was directly proportional to their headacherelieving ability. ${ }^{13}$ A current frequently debated theme in the treatment of this condition is the true importance of vestibular rehabilitation therapy. Various authors have published papers demonstrating the benefits of vestibular rehabilitation therapy in migraine-related auditory-vestibular dysfunction patients. ${ }^{34,39,41,42}$.

\section{FINAL COMMENTS}

Otoneurologists worldwide have studied migrainerelated auditory-vestibular dysfunction, given its clinical manifestations that are similar to those found in a variety of other otoneurological diseases, particularly MS.

Being a recently described syndrome, most otorhinolaryngologists are not prepared for diagnosing this condition, which should be part of the differential diagnosis of vertigo and of the management of migraine patients.

\section{REFERENCES}

1. Sachs OW. Migraine: the evolution of a common disorder. 1st ed. London: Faber \& Faber; 1970.

2. Ménière P. Memoire sur des lesions de loreille interne donnant lieu a des symptomes de congestion cerebrale apoplectiforme. Gaz Med Paris 1861;16:597-601.

3. Gowers WR. The border land of epilepsy: faints, vagal attacks, vertigo, migraine, sleep symptoms, and their treatment. 1st ed. London: Churchill; 1907.

4. Symonds CP. Vertigo. Post Graduate Medical Journal 1926; 1: 6366.

5. Graham JR. Migraine: clinical aspects. In: Vinken PJ, Bruin GW, editors. Handbook of clinical neurology. 1st ed. Amsterdam: Northholland; 1968. p.45-58.

6. Kayan A, Hood JD. Neuro-otological manifestations of migraine. Brain 1984;107(Pt 4):1123-42.

7. Bickerstaff ER. Impairment of consciousness in migraine. Lancet 1961;2:1057-9.

8. Boenheim F. Uber familiare hemicrania vestibularis. Neurologisches Zentralblatt 1917;36:226-9.

9. Heveroch M. La migraine vestibulaire. Rev Neurol 1925;33:925-9.

10. Richter H. Die migraine. In: Bumke O, Foerster O, editors. Handbuch der neurologie. 17 th ed. Berlin: Springer; 1935. p. 166-245.

11. Heyck H. Der kopfschmerz: differentialdiagnostik und therapie fur die praxis. 2nd ed. Stuttgard: G.Thieme; 1958.

12. Wolff HG. Headache and other head pain. 1st ed. New York (NY): Oxford University Press; 1963.

13. Bikhazi PC, Jackson C, Ruckenstein MJ. Efficacy of antimigrainous therapy in the treatment of migraine-associated dizziness. Am J Otol 1997;18(3):350-4

14. Brantberg K, Trees N, Baloh RW. Migraine-associated vertigo. Acta Otolaryngol 2005;125(3):276-9.

15. Neuhauser HK, Lempert T. Diagnostic criteria for migrainous vertigo Acta Otolaryngol 2005;125(11):1247-8.

16. Shepard NT. Differentiation of Ménière's disease and migraineassociated dizziness: a review. J Am Acad Audiol 2006;17(1):69-80.

17. Fedorova ML. The vestibular syndrome in migraine. Klinicheskaya Meditsina 1970;48:70-6.
18. Pearce J. Some aethiological factors in migraine. Em: Cummings JN editors. Background to migraine 4th ed. London: Heinemann Medical 1970. p. 1-7.

19. Kuritzky A, Toglia UJ, Thomas D. Vestibular function in migraine. Headache 1981;21:110-2.

20. Cass SP, Furman JM, Ankerstjerne JKP, Balaban C, Yetiser S, Aydogan B. Migraine-related vestibulopathy. Ann Otol Rhinol Laryngol 1997; 106(3):182-9.

21. Reploeg MD, Goebel JA. Migraine-associated dizziness: patient characteristics and management options. Otol Neurotol 2002; 23(3):36471.

22. Battista RA. Audiometric findings of patients with migraine-associated dizziness. Otol Neurotol 2004; 25(6):987-92.

23. Furman JM, Sparto PJ, Soso M, Marcus D. Vestibular function in migraine-related dizziness: a pilot study. J Vestib Res 2005;15(5-6):327-32.

24. Lempert T, Neuhauser H. Migrainous vertigo. Neurol Clin 2005;23(3):715-30.

25. Radtke A, Lempert T, Neuhauser H. Migraine and Ménière's disease: is there a link? Neurology 2002;59(11):1700-4.

26. Furman JM, Marcus DA. Migrainous vertigo: development of a pathogenetic model and structured diagnostic interview. Curr Opin Neurol 2003;16(1):5-13.

27. Neuhauser H, Lempert T. Vertigo and dizziness related to migraine: a diagnostic challenge. Cephalalgia 2004;24(2):83-91.

28. Lipton RB, Scher AI, Kolodner K, Liberman J, Steiner TJ, Stewart WF. Migraine in the United States: epidemiology and patterns of health care use. Neurology 2002;58(6):885-94.

29. Furman JM, Balaban CD. Migraine-anxiety related dizziness (MARD): a new disorder? J Neurol Neurosurg Psychiatry 2005;76(1):1-8.

30. Olesen, J. The International Headache Society classification and diagnostic criteria are valid and extremely useful. Cephalalgia 1996;16(5):293-5.

31. Ishiyama A, Jacobson KM, Baloh RW. Migraine and benign positional vertigo. Ann Otol Rhinol Laryngol 2000;109(4):377-80.

32. Neuhauser H, Leopold M, von Brevern M, Arnold G, Lempert T. The interrelations of migraine, vertigo, and migrainous vertigo. Neurology 2001;56(4):436-41.

33. Marcus DA, Kapelewski C, Rudy TE, Jacob RG, Furman JM. Diagnosis of migrainous vertigo: validity of a structured interview. Med Sci Monit 2004;10(5):197-201.

34. Gottshall KR, Moore RJ, Hoffer ME. Vestibular rehabilitation for migraine-associated dizziness. Int Tinnitus J 2005;11(1):81-4.

35. von Brevern MD, Zeise D, Neuhauser H, Clarke AH, Lempert $T$. Acute migrainous vertigo: clinical and oculographic findings. Brain 2005;128(Pt 2):365-74.

36. Kolev O. How caloric vestibular irritation influences migraine attacks Cephalalgia 1990;10(4):167-9.

37. Dieterich M, Brandt T. Episodic vertigo related to migraine ( 90 cases): vestibular migraine? J Neurol 1999;246(10):883-92.

38. Dimitri PS, Wall III C, Oas JG, Rauch SD. Application of multivariate statistics to vestibular testing: discriminating between Ménières disease and migraine associated dizziness. J Vestib Res 2001;11(1):53-65.

39. Johnson GD. Medical management of migraine-related dizziness and vertigo. Laryngoscope 1998;108(1 Pt 2):1-28.

40. Swartz R, Longwell P. Treatment of vertigo. Am Fam Physician 2005;71(6):1115-22.

41. Whitney SL, Wrisley DM, Furman JM. Physical therapy for migrainerelated vestibulopathy and vestibular dysfunction with history of migraine. Laryngoscope 2000;110(9):1528-34.

42. Wrisley DM, Whitney SL, Furman JM. Vestibular rehabilitation outcomes in patients with a history of migraine. Otol Neurotol 2002;23(4):483-7.

43. Hinchcliffe R. The dizzy patient. J Iowa Med Soc 1963;53:667-78.

44. Eklund S. Headache in Ménières disease. Auris Nasus Larynx 1999;26(4):427-33.

45. Ceranic B, Luxon L. Ménières-migraine overlap syndrome. Proceedings of the 29th Mid-Winter American Research in Otolaryngology 
ARO; 2006 feb 5-7; Baltimore, MD, USA.

46. Kentala E, Pyykko I. Benign recurrent vertigo--true or artificial diagnosis? Acta Otolaryngol 1997;529(Suppl 1):101-3.

47. Baloh RW. The dizzy patient. Postgrad Med 1999;105(2):161-4.

48. Atkinson M. Ménières syndrome and migraine: observations on common causal relationship. Ann Intern Med 1943;18:797-808.
49. Olsson JE. Neurotologic findings in basilar migraine. Laryngoscope 1991;101(1 Pt 2 Suppl 52):1-41.

50. Baloh RW. Neurotology of migraine. Headache 1997;37(10):615-21.

51. Baloh RW. Episodic vertigo: central nervous system causes. Curr Opin Neurol 2002;15(1):17-21. 